\title{
Hospital-acquired hyperkalaemia
}

\author{
Neil CM Bacon
}

A 56-year-old man was admitted to his local hospital complaining of pleuritic chest pain and shortness of breath. He was known to the regional renal unit with stable chronic renal failure of unknown aetiology (bilateral small kidneys on ultrasound examination) and hypertension. Two months before admission his creatinine had been $775 \mu \mathrm{mol} / 1$ and serum potassium $5.6 \mathrm{mmol} / 1$. $\mathrm{He}$ was taking the following medication: erythropoietin, alfacalcidol, calcium carbonate, isosorbide mononitrate, aspirin and atenolol.

Pulmonary embolism was felt to be the most likely diagnosis and he was commenced on intravenous heparin at 1000 units/h after a bolus dose of 5000 units. This was continued during his 10 days in hospital. A 'renal' diet was ordered.

Serum potassium and creatinine during his admission are shown in the table. He received three doses of a potassium exchange resin (Calcium resonium) on day three, this was repeated on day eight. Fluid intake and urine output were good throughout the hospital stay.

Nuffield Department of Medicine, John

Radcliffe Hospital, Oxford, UK

NCM Bacon

Correspondence to $\mathrm{Dr}$ NCM Bacon, October

Cottage, Stonesfield,

Oxfordshire OX8 8PW,

UK

Accepted 29 March 1996

Table Serum potassium and creatinine

\begin{tabular}{lllllllll}
\hline Day & 1 & 3 & 4 & 5 & 6 & 8 & 9 & 10 \\
\hline Potassium $(\mathrm{mmol} / \mathrm{l})$ & 5.6 & 5.3 & 5.1 & 5.4 & 5.3 & 6.4 & 6.0 & 6.0 \\
Creatinine $(\mu \mathrm{mol} / \mathrm{l})$ & 786 & & 780 & & 752 & 765 & 746 & 756 \\
\hline
\end{tabular}

\section{Questions}

1 What is the cause of the increase in serum potassium levels?

2 Give three factors putting this patient at increased risk of hyperkalaemia.

3 Which other group of patients is particularly vulnerable to such an effect? 
Answers

\section{QUESTION 1}

Heparin-induced suppression of aldosterone synthesis. The clinical diagnosis was confirmed by a plasma aldosterone level on day eight of $180 \mathrm{pmol} / \mathrm{l}$, inappropriately low in the presence of hyperkalaemia.

\section{QUESTION 2}

Chronic renal insufficiency, nonsteroidal antiinflammatory drugs (NSAIDs), beta-blockers.

\section{QUESTION 3}

Patients with diabetes mellitus, especially those on angiotensin-converting enzyme (ACE) inhibitors.

\section{Discussion}

Hyperkalaemia is a potent stimulus to aldosterone release: a rise in serum potassium of as little as $0.1 \mathrm{mmol} / 1$ results in a $35 \%$ increase in aldosterone levels in normal individuals. ${ }^{1}$ This acts on the distal tubules of the kidney to increase potassium secretion and ensures that serum potassium levels are tightly maintained in a narrow range.

Therapeutic doses of heparin decrease secretion of aldosterone from the adrenals in both health and disease states. ${ }^{2}$ The mechanism by which this occurs is poorly understood but is believed to involve selective inhibition of 18-hydroxylase, the enzyme involved in the final step of aldosterone synthesis in the zona glomerulosa. Experimentally, rats treated with prolonged courses of heparin show selective atrophy of the zona glomerulosa with preservation of the other regions of the adrenals. ${ }^{3}$ Healthy individuals treated with heparin will not normally develop hyperkalaemia as decreased aldosterone synthesis stimulates increased renin production, preventing the development of electrolyte disturbances. ${ }^{2}$

The situation is different in patients with renal impairment. As glomerular filtration rate falls the ability of the kidney to excrete a potassium load is diminished. The resultant increase in serum potassium stimulates aldosterone release which increases potassium secretion in the remaining nephrons. Thus many patients with chronic renal disease are dependent on hyperaldosteronism to maintain normokalaemia. ${ }^{4}$ In such patients, any fall in aldosterone levels can result in marked hyperkalaemia.

1 Himathongkam T, Dluhy RG, Williams, GH. Potassiumaldosterone-renin interrelationships. 7 Clin Endocrinol Metab aldosterone-renin

2 O'Kelly R, Maga F, McKenna TJ. Routine heparin therapy inhibits adrenal aldosterone production. $f$ Clin Endocrinol inhibits adrenal aldostero

Metab 1983; 56: 108-12.
Abbott EC, Monkhouse FC, Steiner JW, Laidlaw JC. Effect of a sulfated mucopolysaccharide (R01-8307) on the zona glomerulosa of the rat adrenal gland. Endocrinology 1966 78: $651-4$.

4 Berl T, Katz FH, Henrich WL, Torrente AD, Schrier RW. Role of aldosterone in the control of sodium excretion in patients with advanced chronic renal failure. Kidney Int 1978; 14: $228-35$.

5 Romero JC, Dunlap CL, Strong CG. The effect of indomethacin and other anti-inflammatory drugs on the renin-angiotensin system in man. $\mathcal{F}$ Clin Invest 1976; 58: $282-8$.

\section{Iatrogenic causes of hyperkalaemia}

- spurious - haemolysis during venesection, contamination by intravenous fluids

- administration - parenteral infusions, transfusion of stored blood

- drugs - potassium-sparing diuretics, ACEinhibitors, beta-blockers, NSAIDs, heparin

In addition to serum potassium levels, aldosterone synthesis is dependent on the integrity of the renin-angiotensin system. Both NSAIDs ${ }^{5}$ and beta-blockers ${ }^{6}$ are known to inhibit the release of renin from the kidney. Aspirin and atenolol in this patient's medication thus increased his sensitivity to hyperkalaemia following heparin-induced inhibition of aldosterone synthesis.

Patients with diabetes mellitus are known to be at risk of hyperkalaemia through a number of mechanisms, including insulin deficiency, renal impairment and drug effects. ${ }^{7}$ Hyporeninaemic hypoaldosteronism has increasingly been recognised as an additional factor increasing the risk of hyperkalaemia in diabetes. ${ }^{8}$ Autonomic dysfunction and mild renal impairment are both probably involved in the hyporeninaemia of diabetes. Such patients may have normal or slightly raised potassium levels in the steady state but can develop marked hyperkalaemia when treated with therapeutic doses of heparin. ${ }^{9}$

ACE inhibitors prevent the generation of angiotensin II and thus reduce the stimulus to aldosterone synthesis. Patients taking ACE inhibitors, especially if they have diabetes and/ or renal dysfunction, may become hyperkalaemic with the institution of heparin therapy. ${ }^{10}$

In addition to its role in the treatment and prevention of venous thromboembolism, heparin is increasingly used in patients with coronary or peripheral vascular disease. Many of those requiring such treatment will be patients with renal disease and diabetes. It is important that clinicians prescribing heparin are aware of its possible effects on potassium balance in such patients.

\section{Final diagnosis}

Heparin-induced suppression of aldosterone synthesis

Keywords: hyperkalaemia, heparin, hypoaldosteronism

6 Leonetti G, Mayer G, Morganti A, et al. Hypotensive and renin suppressing activities of propranolol in hypertensive patients. Clin Sci Mol Med 1975; 48: 491-9.

7 Jarman PR, Kehely AM, Mather HM. Hyperkalaemia in diabetes: prevalence and associations. Postgrad Med f 1995 71: $551-2$.

8 Tuck ML, Sambhi MP, Levin L. Hyporeninemic hypoaldosteronism in diabetes mellitus. Diabetes 1979; 28: $237-$ 41.

9 Phelps KR, Oh MS, Carroll HJ. Heparin-induced hyperkalemia: report of a case. Nephron 1980; 25: 254-8

10 Durand D, Ader J-L, Rey J-P, et al. Inducing hyperkalaemia by converting enzyme inhibitors and heparin. Kidney Int 1988; 34 (suppl 25): S196-7. 